Moroccan J. of Pure and Appl. Anal. (MJPAA)

Volume 6(2), 2020, Pages 255-265

ISSN: Online 2351-8227 - Print 2605-6364

DOI: $10.2478 / \mathrm{mjpaa}-2020-0020$

\title{
Sum of the spaces on ordered setting
}

\section{T. M. Al-ShAMI}

\begin{abstract}
Aвstract. One of the divergences between topology and ordered topology is that some topological concepts such as separation axioms and continuous maps are defined using open neighborhoods or neighborhoods without any difference, however, they are distinct on the ordered topology according to the neighborhoods: Are they open neighborhoods or not? In this paper, we present the concept of sum of the ordered spaces using pairwise disjoint topological ordered spaces and study main properties. Then, we introduce the properties of ordered additive, finitely ordered additive and countably ordered additive which associate topological ordered spaces with their sum. We prove that the properties of being $T_{i}$-ordered and strong $T_{i}$-ordered spaces are ordered additive, however, the properties of monotonically compact and ordered compact spaces are finitely ordered additive. Also, we define a mapping between two sums of the ordered spaces using mappings between the ordered spaces and deduce some results related to some types of continuity and homeomorphism. We complete this work by determining the conditions under which a topological ordered space is sum of the ordered spaces.
\end{abstract}

Mathematics Subject Classification (2020): 54A05, 54C08, 54D99, 54F05, 54F65.

Key words and phrases: Sum of the ordered spaces, Additive ordered property, $T_{i}$-ordered space, Monotonically compact space, Continuity.

\section{Introduction}

In 1965, Nachbin [28] initiated the concept of topological ordered spaces by adding a partial order relation to the structure of a topological space. Both topology and partial order

Received : 10 June 2020 - Accepted: 14 September 2020.

(C) This article is published with open access by Sidi Mohamed Ben Abdallah University.

The Author: Department of Mathematics, Sana'a University, Sana'a, Yemen

e-mail: tareqalshami83@gmail.com. 
relation are defined as independent each of other. Nachbin [28] proved some results concerning $T_{i}$-ordered spaces $(i=2,4)$ and compactness, which generalized well-known theorems for topological spaces. Also, he defined convex spaces and completely regular ordered spaces and studied some of their properties. In 1966, McCartan [24] studied some properties of subsets of a partially ordered set. In 1968, he (see [25]) introduced $T_{i}$-separation axioms $(i=0,1,2,3,4)$ on topological ordered spaces and proved some results concerning $T_{i}$-ordered spaces $(i=2,3)$ and local compactness. Burgess and McCartan [13] introduced a concept of order-connectedness and compared four procedures for defining the order-continuous functions between topological ordered spaces. In 1977, Burgess and Fitzpatrick [12] investigated the consequences of $T_{i}$-ordered spaces under the conditions of convexity, continuity, anticontinuity and bicontinuity of a topological ordered spaces which given in [26].

It worthily noted that the concepts of $T_{i}$-spaces and continuous maps are defined using open neighborhoods or neighborhoods without any difference, however, the counterparts of these concepts on the ordered topology are distinct according to the type of neighborhoods: Are they open neighborhoods or not?

In 1991, Arya and Gupta [11] investigated some new ordered separation axioms, namely semi $T_{1}$-ordered and semi $T_{2}$-ordered spaces. These two concepts were generalized by [23] in 2002. In another direction of study ordered spaces, Popescu [29] studied separation axioms by replacing a partial order relation by an arbitrary binary relation. Then, Mendez et al. [27] studied separation axioms by replacing the identity relation on topological space by a specific binary relation. In 2002, Kumar [18] studied the concepts of continuous, open, closed and homeomorphism mappings between topological ordered spaces and studied their main properties. These concepts were generalized between supra topological ordered spaces in [1]. In 2004, Das [14] presented some ordered separation axioms in ordered spaces and highlighted on their properties on supra topological ordered spaces. El-Shafei et al. [15] completed Das's work by studying strong separation axioms on supra topological ordered spaces. Some significant results related to completely regular ordered and strictly completely regular ordered spaces were investigated in [19, 21]. In 2005, Künzi and Richmond [20] presented an explicit construction of the $T_{0}$-ordered reflection of an ordered topological space and characterized ordered topological spaces whose $T_{0}$-ordered reflection is $T_{1}$-ordered. In 2014, Lazaar and Mhemdi [22] investigated some properties of $T_{0}$-ordered reflection. In 2018, Shanthi and Rajesh [30] introduced new types of $T_{i}$-ordered spaces, namely $\omega T_{i}$-ordered $(i=1,2)$ and $\omega$-regularly ordered spaces.

To study topological spaces on ordered setting, I and my co-authors defined the concepts of soft topological ordered in [6] and supra soft topological ordered spaces in [3]. In these two works, we introduced the concept of increasing and decreasing soft sets and utilized it to explored some types of soft separation axioms. Then we $[4,7,8,9,16]$ initiated some types of ordered maps and probed their main properties. Recently, we [5] have established two types of ordered soft separation axioms and compared between them with the help of examples.

This paper was organized as follows: In Section 2, we recall some definitions and results of topological ordered spaces and sum of the spaces that will help us to understand this work. In Section 3, we introduce the concept of sum of the ordered spaces using pairwise disjoint topological ordered spaces. Also, we define ordered additive, finitely ordered additive and 
countably ordered additive properties, and give some examples of these properties such as $T_{i^{-}}$ ordered and monotonically compact spaces. Section 4 concludes the paper and future works.

\section{Preliminaries}

This section presents the definitions and properties related to partial order relations, topological ordered spaces and sum of the spaces that will be needed in the sequels.

Definition 2.1. ([17]). A binary relation $R$ on a nonempty set $Y$ is called:

(i): reflexive if $(y, y) \in R$ for each $y \in Y$,

(ii): anti-symmetric if $(y, z),(z, y) \in R$ implies that $y=z$,

(iii): transitive if $(y, z),(z, x) \in R$ implies that $(y, x) \in R$,

(iv): a partial order relation if it is reflexive, anti-symmetric and transitive.

The diagonal relation on any non-empty set $Y$, which given by $\{(y, y): y \in Y\}$ shall be shortly denoted by $\triangle$.

Throughout this paper, we denote a partial order relation by $\preceq$ or $\leq$.

Definition 2.2. ([28]). Let $X$ be a subset of a partially ordered set $(Y, \preceq)$ and $y \in Y$. Then:

(i): $i(y)=\{a \in Y: y \preceq a\}$ and $d(y)=\{a \in Y: a \preceq y\}$.

(ii): $i(X)=\bigcup\{i(a): a \in X\}$ and $d(X)=\bigcup\{d(a): a \in X\}$.

(iii): $A$ set $X$ is called increasing (resp. decreasing) if $X=i(X)($ resp. $X=d(X))$.

Definition 2.3. ([2]). A subset $A$ of $(Y, \preceq)$ is called monotonic if $A$ is increasing or decreasing. In other words, $A=i(A)$ or $A=d(A)$.

Definition 2.4. ([28]). A triple $(Y, \tau, \preceq)$ is said to be a topological ordered space, where $(Y, \preceq)$ is a partially ordered set and $(Y, \tau)$ is a topological space.

Henceforth, $(Y, \tau, \preceq)$ and $(Z, \theta, \leq)$ denote topological ordered spaces.

Definition 2.5. ([25]). Let $A$ be a subset of $(Y, \tau, \preceq)$. We define a topological ordered subspace $\left(A, \tau_{A}, \preceq_{A}\right)$ of $(Y, \tau, \preceq)$ as follows $\tau_{A}=\{A \cap G:$ for each $G \in \tau\}$ and $\preceq_{A}=\preceq \bigcap A \times A$.

Definition 2.6. A subset $A$ of $(Y, \tau, \preceq)$ is called:

(i): a neighborhood of $y \in Y$ if there exists an open set $U$ such that $y \in U \subseteq A$.

(ii): an open neighborhood of $y \in Y$ if it is an open set containing $y$.

(iii): an increasing neighborhood of $y \in Y$ if it is a neighborhood of $y \in Y$ and $i(A)=A$.

(iv): a decreasing neighborhood of $y \in Y$ if it is a neighborhood of $y \in Y$ and $d(A)=A$.

Definition 2.7. ([25]). ( $Y, \tau, \preceq)$ is said to be:

(i): lower $T_{1}$-ordered if for each $x \npreceq y \in Y$, there is an increasing neighborhood $U$ of $x$ such that $y \notin U$.

(ii): upper $T_{1}$-ordered if for each $x \npreceq y \in Y$, there is a decreasing neighborhood $U$ of $y$ such that $x \notin U$.

(iii): $T_{0}$-ordered if it is lower $T_{1}$-ordered or upper $T_{1}$-ordered.

(iv): $T_{1}$-ordered if it is lower $T_{1}$-ordered and upper $T_{1}$-ordered.

(v): $T_{2}$-ordered if for each $x \npreceq y \in Y$, there are disjoint neighborhoods $U$ and $V$ of $x$ and $y$, respectively, such that $U$ is increasing and $V$ is decreasing. 
(vi): regular ordered if for every increasing (resp. decreasing) closed set $F$ and $y \in Y$ such that $y \notin F$, there are disjoint increasing (resp. decreasing) neighborhood $U$ and decreasing (resp. increasing) neighborhood $V$ such that $F \subseteq U$ and $y \in V$.

(vii): normal ordered if for every two disjoint closed sets $F$ and $H$ such that $F$ is increasing and $H$ is decreasing, there are two disjoint increasing neighborhood $U$ and decreasing neighborhood $V$ such that $F \subseteq U$ and $H \subseteq V$.

(viii): $T_{3}$-ordered (resp. $T_{4}$-ordered) if it is both $T_{1}$-ordered and regular ordered (resp. normal ordered).

McCartan [25] also introduced the concepts of strong $T_{i}$-ordered spaces by replacing the word neighborhood by open set in the above definition.

Definition 2.8. ([2]). The collection $\left\{G_{j}: j \in J\right\}$ of open subsets of $(Y, \tau, \preceq)$ is called a monotonically open cover of $Y$ provided that $Y=\bigcup_{j \in J} G_{j}$ and all $G_{j}$ are monotonic.

Definition 2.9. ([2]). ( $Y, \tau, \preceq)$ is said to be:

(1) monotonically compact provided that every monotonically open cover of $Y$ has a finite subcover.

(2) ordered compact if every soft open cover of $Y$ has a finitely monotonic subcover.

Proposition 2.1. [2] Every monotonically closed (resp. closed) subset of a monotonically compact (resp. an ordered compact) space $(Y, \tau, \preceq)$ is monotonically compact (resp. ordered compact).

Definition 2.10. ([13]). ( $Y, \tau, \preceq)$ is said to be:

(1) order-separated if there are disjoint closed sets $A, B$ in $X$ such that $A$ is increasing, $B$ is decreasing and $A \cup B=Y$.

(2) order-connected if and only if it is not order-separated.

Definition 2.11. ([18]). $f_{\phi}:(Y, \tau, \preceq) \rightarrow(Z, \theta, \leq)$ is called:

(i): I (resp. D, B)-continuous if and only if the inverse image of each open set is increasing (resp. decreasing, balancing) open.

(ii): soft I (resp. D, B) -open if the image of every open is increasing (resp. decreasing, balancing) open.

(iii): soft I (resp. D, B) -closed if the image of every closed is increasing (resp. decreasing, balancing) closed.

Definition 2.12. ([18]). A bijective soft map $g:(Y, \tau, \preceq) \rightarrow(Z, \theta, \leq)$ is called I (resp. D, B) homeomorphism if it is I-continuous and I-open (resp. D-continuous and D-open, B-continuous and B-open).

Definition 2.13. ([17]). Let $\left\{\left(Y_{i}, \tau_{i}\right): i \in I\right\}$ be a family of pairwise disjoint topological spaces and $Y=\bigcup_{i \in I} Y_{i}$. Then the collection

$$
\tau=\left\{G \subseteq X: G \cap Y_{i} \text { is an open set in }\left(Y_{i}, \tau_{i}\right) \text { for every } i \in I\right\}
$$

defines a topology on $Y$.

This topological space is called sum of the spaces and denoted by $\left(\oplus_{i \in I} Y_{i}, \tau\right)$.

Recently, the concept of sum of the spaces has been introduced and studied on soft setting by [10].

Proposition 2.2. ([17]). All sets $Y_{i}$ are clopen subsets of $\left(\oplus_{i \in I} Y_{i}, \tau\right)$. 


\section{Sum of topological ordered spaces}

In this section, we present the concept of sum of topological spaces on ordered setting and then we define ordered additive, finitely ordered additive and countably ordered additive properties. We discuss some concepts in terms of these properties with the help of illustrative examples.

Proposition 3.1. Let $\left\{\left(Y_{i}, \tau_{i}, \preceq_{i}\right): i \in I\right\}$ be a family of pairwise disjoint topological ordered spaces. Then $(Y, \tau, \preceq)$ is a topological ordered space, where

(1) $Y=\bigcup_{i \in I} Y_{i}$;

(2) $\tau=\left\{U\right.$ over $\bigcup_{i \in I} Y_{i}: U \cap Y_{i} \in \tau_{i}$ for every $\left.i \in I\right\}$ and

(3) $\preceq=\bigcup_{i \in I} \preceq_{i}$.

Proof. It follows from Proposition 2.13 that $\left(Y_{i}, \tau_{i}, A\right)$ is a topological space. It remains to prove that $\preceq$ is a partial order relation on $Y$. It is clear that $\preceq$ is reflexive on $Y$. Since $\preceq_{i}$ is antisymmetric and transitive for each $i$ and $\preceq_{i} \cap \preceq_{j}=\varnothing$ for each $i \neq j$, then $\preceq$ is anti-symmetric and transitive on $Y$. Thus, $\preceq$ is a partial order relation on $Y$. Hence, $(Y, \tau, \preceq)$ is a topological ordered space.

Definition 3.1. The topological ordered space $(Y, \tau, \preceq)$ given in the above proposition is said to be the sum of topological ordered spaces and is denoted by $\left(\oplus_{i \in I} Y_{i}, \tau, \preceq\right)$.

Proposition 3.2. If $U$ is a monotonic subset of $\left(\oplus_{i \in I} Y_{i}, \preceq\right)$, then $U \cap Y_{i}$ is a monotonic subset of $\left(Y_{i}, \preceq_{i}\right)$ for every $i \in I$.

Proof. Necessity: Let $U$ be a monotonically subset of $\left(\oplus_{i \in I} Y_{i}, \preceq\right)$. Say, it is increasing. Suppose that there exists $i \in I$ such that $U \cap Y_{i}$ is not an increasing subset of $\left(Y_{i}, \preceq_{i}\right)$. Then there exists $y \in Y_{i}$ such that $y \notin U \cap Y_{i}$ and $x \preceq_{i} y$ for some $x \in U \cap Y_{i}$. Since $Y_{i} \cap Y_{j}=\varnothing$ for each $i \neq j$, then $U$ is not increasing. We obtain a contradiction.

The converse of the above proposition is not always true as it is illustrated in the following example.

Example 3.1. Let $\preceq_{1}=\triangle \bigcup\left\{\left(x_{2}, x_{1}\right)\right\}$ and $\preceq_{2}=\triangle \bigcup\left\{\left(y_{1}, y_{2}\right)\right\}$ be two partial order relations on $X=\left\{x_{1}, x_{2}\right\}$ and $Y=\left\{y_{1}, y_{2}\right\}$, respectively. Now, $U=\left\{x_{1}, y_{1}\right\}$ is neither decreasing nor increasing subset of $(X \cup Y, \preceq)$. Therefore it is not monotonic. However, $U \cap X=\left\{x_{1}\right\}$ is an increasing subset of $\left(X, \preceq_{1}\right)$ and $U \cap Y=\left\{y_{1}\right\}$ is a decreasing subset of $\left(Y, \preceq_{2}\right)$.

Proposition 3.3. A subset $U$ of $\left(\oplus_{i \in I} Y_{i}, \preceq\right)$ is increasing (resp. deceasing) if and only if all sets $U \cap Y_{i}$ are increasing (resp. deceasing) in $\left(Y_{i}, \preceq_{i}\right)$.

Proof. The necessary condition follows from Proposition 3.2.

To prove the sufficient condition, let $U \cap Y_{i}$ be an increasing (resp. a deceasing) subset of $\left(Y_{i}, \preceq_{i}\right)$ for every $i \in I$. Since $Y_{i} \cap Y_{j}=\varnothing$ for each $i \neq j$ and $\preceq=\bigcup_{i \in I} \preceq_{i}$, then $U$ is an increasing (resp. a deceasing) subset of $\left(\oplus_{i \in I} Y_{i}, \preceq\right)$.

Proposition 3.4. All sets $Y_{i}$ are monotonically clopen in $\left(\oplus_{i \in I} Y_{i}, \tau, \preceq\right)$.

Proof. It follows from Proposition 2.2 and Proposition 3.3. 
Corollary 3.1. Every sum of topological ordered spaces is order-separated.

Proposition 3.5. If $\left\{\left(Y_{i}, \tau_{i}, \preceq_{i}\right): i \in I\right\}$ is a class of pairwise disjoint topological ordered spaces and $X_{i}$ is a subspace of $Y_{i}$ for every $i \in I$, then the ordered topology of the sum of subspaces $\left\{\left(X_{i}, \tau_{X_{i}}, \preceq_{X_{i}}\right.\right.$ )$: i \in I\}$ and the topological ordered subspace on $\bigcup_{i \in I} X_{i}$ of the sum topology $\left(\oplus_{i \in I} Y_{i}, \tau, \preceq\right)$ coincide.

Proof. Straightforward.

Definition 3.2. A property $\mathcal{P}$ is said to be:

(1) ordered additive if for any family of topological ordered spaces $\left\{\left(Y_{i}, \tau_{i}, \preceq_{i}\right): i \in I\right\}$ with the property $\mathcal{P}$, the sum of this family also has property $\mathcal{P}$.

(2) finitely ordered additive (resp., countably ordered additive) if for any finite (resp., countable) family topological ordered spaces with the property $\mathcal{P}$, the sum of this family also has property $\mathcal{P}$.

Lemma 3.1. $U \cap Y_{i}$ is increasing (resp. deceasing) neighborhood of an element in $\left(Y_{i}, \tau_{i}, \preceq_{i}\right)$ iff it is increasing (resp. deceasing) neighborhood of this element in $\left(\oplus_{i \in I} Y_{i}, \tau, \preceq\right)$.

Proof. Necessity: Let $U \cap Y_{i}$ be a neighborhood of $y \in Y_{i}$. Then there is an open subset $G$ of $\left(Y_{i}, \tau_{i}, \preceq_{i}\right)$ such that $y \in G \subseteq U \cap Y_{i}$. By Definition 3.1, we obtain $G \in \tau$. Then $U \cap Y_{i}$ is a neighborhood of $y$ in $\left(\oplus_{i \in I} Y_{i}, \tau, \preceq\right)$. Since $U \cap Y_{i}$ is increasing (resp. deceasing) in $\left(Y_{i}, \tau_{i}, \preceq_{i}\right)$, then it follows from Proposition 3.3 that $U \cap Y_{i}$ is increasing (resp. deceasing) in $\left(\oplus_{i \in I} Y_{i}, \tau, \preceq\right)$. This finishes the proof the necessary condition.

Sufficiency: It can be proved by following similar arguments given in the proof of the necessary part.

Theorem 3.1. The property of being a $T_{i}$-ordered space is an ordered additive property for $i=0,1,2,3,4$.

Proof. We prove the theorem in the case of $i=2$. Let $y \npreceq z \in \oplus_{i \in I} Y_{i}$. Then we have the following two cases:

1. There exists $i_{0} \in I$ such that $y, z \in Y_{i_{0}}$.

Since $\left(Y_{i_{0}}, \tau_{i_{0}}, \preceq_{i_{0}}\right)$ is $T_{2}$-ordered, then there exist disjoint increasing neighborhood $U$ of $y$ and decreasing neighborhood $V$ of $z$ in $\left(Y_{i_{0}}, \tau_{i_{0}}, \preceq_{i_{0}}\right)$. It follows from the above lemma that $U$ is increasing neighborhood of $y$ and $V$ is decreasing neighborhood of $z$ in $\left(\oplus_{i \in I} Y_{i}, \tau, \preceq\right)$ such that $U \cap V=\varnothing$.

2. There exist $i_{0} \neq j_{0} \in I$ such that $y \in Y_{i_{0}}$ and $z \in Y_{j_{0}}$.

Now, $Y_{i_{0}}$ is an increasing neighborhood of $y$ and $Y_{j_{0}}$ is a decreasing neighborhood of $z$. Obviously, $Y_{i_{0}}$ and $Y_{j_{0}}$ are disjoint.

It follows from the two cases above that $\left(\oplus_{i \in I} Y_{i}, \tau, \preceq\right)$ is a $T_{2}$-ordered space.

The theorem can be proved similarly in the cases of $i=0,1$.

To prove the theorem in the cases of $i=3$ and $i=4$, it suffices to prove the regularity ordered and normality ordered, respectively.

First, we prove the regularity ordered property. Let $F$ be an increasing closed subset of $\left(\oplus_{i \in I} Y_{i}, \tau, \preceq\right)$ such that $y \notin F$. It follows from Proposition 3.2, that $F \cap Y_{i}$ is increasing closed in $\left(Y_{i}, \tau_{i}, \preceq_{i}\right)$ for each $i \in I . y \in \oplus_{i \in I} Y_{i}$ implies that there is only $i_{0} \in I$ such that $y \in Y_{i_{0}}$. Therefore there are disjoint neighborhoods $U$ of $F \cap Y_{i_{0}}$ and $V$ of $y$ in $\left(Y_{i_{0}}, \tau_{i_{0}}, \preceq_{i_{0}}\right)$ such that $U$ is increasing and $V$ is decreasing. Now, $U \underset{i \neq i_{0}}{U} Y_{i}$ is an increasing neighborhood of $F$ in 
$\left(\oplus_{i \in I} Y_{i}, \tau, \preceq\right)$. The disjointness between $U \bigcup_{i \neq i_{0}} Y_{i}$ and $V$ ends the proof that $\left(\oplus_{i \in I} Y_{i}, \tau, \preceq\right)$ is a regular ordered space.

Second, we prove the normality ordered property. Let $F$ and $H$ be two disjoint closed subsets of $\left(\oplus_{i \in I} Y_{i}, \tau, \preceq\right)$ such that $F$ is increasing and $H$ is decreasing. It follows from Proposition 3.3, that $F \cap Y_{i}$ and $H \cap Y_{i}$ are closed in $\left(Y_{i}, \tau_{i}, \preceq_{i}\right)$ for each $i \in I$ such that $F \cap Y_{i}$ is increasing and $H \cap Y_{i}$ is decreasing. Since $\left(Y_{i}, \tau_{i}, \preceq_{i}\right)$ is normal ordered for each $i \in I$, then there exist disjoint neighborhoods $U_{i}$ of $F \cap Y_{i}$ and $V_{i}$ of $H \cap Y_{i}$ such that $U_{i}$ is increasing and $V_{i}$ is decreasing in $\left(Y_{i}, \tau_{i}, \preceq_{i}\right)$. Therefore $F \subseteq \bigcup_{i \in I} U_{i}, H \subseteq \bigcup_{i \in I} V_{i}$ and $\left[\bigcup_{i \in I} U_{i}\right] \cap\left[\bigcup_{i \in I} V_{i}\right]=\varnothing$. Hence, $\left(\oplus_{i \in I} Y_{i}, \tau, \preceq\right)$ is normal ordered space.

Theorem 3.2. The property of being a strong $T_{i}$-ordered space is an ordered additive property for $i=0,1,2,3,4$.

Proof. The proof is similar to that of Theorem 3.1.

Proposition 3.6. The property of being a monotonically compact space is a finitely ordered additive property.

Proof. Let $\left\{\left(Y_{k}, \tau_{k}, \preceq_{k}\right): k \in\{1,2, \ldots, n\}\right\}$ be a finite family of pairwise disjoint monotonically compact spaces and let $\left(\oplus_{k=1}^{n} Y_{k}, \tau, \preceq\right)$ be the sum of this family. Suppose that $\left\{U_{i}: i \in I\right\}$ is an open cover of $Y=\bigcup_{k=1}^{n} Y_{k}$. Then $Y_{k}=\bigcup_{i \in I}\left[U_{i} \cap Y_{k}\right]$ for every $k \leq n$. Since $\left(Y_{k}, \tau_{k}, \preceq_{k}\right)$ is monotonically compact for every $k \leq n$, then there exist finite subsets $M_{1}, M_{2}, \ldots, M_{n}$ of $I$ such that $Y_{1}=\bigcup_{i \in M_{1}}\left[U_{i} \cap Y_{1}\right]$ and all $U_{i} \cap Y_{1}$ are monotonic, $Y_{2}=\bigcup_{i \in M_{2}}\left[U_{i} \cap Y_{2}\right]$ and all $U_{i} \cap Y_{2}$ are monotonic, ..., $Y_{n}=\bigcup_{i \in M_{n}}\left[U_{i} \cap Y_{n}\right]$ and all $U_{i} \cap Y_{n}$ are monotonic. Letting $M=\bigcup_{k=1}^{n} M_{k}$. Now, $Y=\bigcup_{i \in M}\left[U_{i} \cap Y_{k}\right]$ for every $k \leq n$. Since $M$ is finite and all $U_{i} \cap Y_{k}$ are monotonic, then $\left(\oplus_{k=1}^{n} Y_{s}, \tau, \preceq\right)$ is monotonically compact.

Proposition 3.7. The property of being an ordered compact space is a finitely ordered additive property. Proof. The proof is similar to that of Proposition 3.6.

The following example shows that the properties of monotonically compactness and ordered compactness are not ordered additive.

Example 3.2. Let $X_{n}=\{2 n-1,2 n\}$, where $n$ belongs to the set of natural numbers $\mathbb{N}$. Consider $\tau_{n}$ is the discrete topology and $\preceq_{n}$ is the equality relation on $X_{n}$ for each $n$. Now, $\left\{\left(X_{n}, \tau_{n}, \preceq_{n}\right): n \in \mathcal{N}\right\}$ is a family of pairwise disjoint monotonically compact and ordered compact spaces. Obviously, $\preceq$ is the equality relation on $X$ and the sum of these spaces $\left(\oplus_{n \in \mathcal{N}} Y_{n}, \tau, \preceq\right)$ is discrete. Then $\left(\oplus_{n \in \mathcal{N}}, \tau, \preceq\right)$ is neither monotonically compactness neither ordered compactness. Hence, the properties of monotonically compactness and ordered compactness are not ordered additive.

Proposition 3.8. If the sum of topological ordered spaces $\left(\oplus_{i \in I} Y_{i}, \tau, \preceq\right)$ is monotonically compact (resp. ordered compact), then the following two assertions are true:

(1) all $\left(Y_{i}, \tau_{i}, \preceq_{i}\right)$ are monotonically compact (resp. ordered compact). 
(2) the index set I is finite.

Proof. 1. It follows from Corollary $3.4,\left(Y_{i}, \tau_{i}, \preceq_{i}\right)$ is a monotonically closed subspace of $\left(\oplus_{i \in I} Y_{i}, \tau, \preceq\right.$ ) for each $i \in I$. It follows from Proposition 2.1 that $\left(Y_{i}, \tau_{i}, \preceq_{i}\right)$ is monotonically compact (ordered compact) for each $i \in I$.

2. Let $\left(\oplus_{i \in I} Y_{i}, \tau, \preceq\right)$ be the sum of topological ordered spaces. Then $\Lambda=\left\{Y_{i}: i \in I\right\}$ is a (monotonically) open cover of $Y=\bigcup_{i \in I} Y_{i}$. It is clear that $\Lambda$ does not have a finite subcover if $I$ is infinite. This contradicts the fact that $\left(\oplus_{i \in I} Y_{i}, \tau, \preceq\right)$ is ordered (monotonically) compact. Hence, it must be that $I$ is finite.

Similarly to the proof of Proposition 3.6, one can prove the following result.

Proposition 3.9. The property of being a monotonically Lindelöf (ordered Lindelöf) space is a countably additive property.

Definition 3.3. Let $\left\{f_{i}:\left(Y_{i}, \tau_{i}, \preceq_{i}\right) \rightarrow\left(Z_{i}, \theta_{i}, \leq_{i}\right): i \in I\right\}$ be a family of mappings. Then we define a mapping $f:\left(\oplus_{i \in I} Y_{i}, \tau, \preceq\right) \rightarrow\left(\oplus_{i \in I} Z_{i}, \theta, \leq\right)$ as follows: For each subsets $A$ and $B$ of $\left(\oplus_{i \in I} Y_{i}, \tau, \preceq\right)$ and $\left(\oplus_{i \in I} Z_{i}, \theta, \leq\right)$, respectively, we have:

(1) $f(A)=\bigcup_{i \in I} f_{i}\left(A \cap Y_{i}\right)$; and

(2) $f^{-1}(B)=\bigcup_{i \in I} f_{i}^{-1}\left(B \cap Z_{i}\right)$

Theorem 3.3. A mapping $f:\left(\oplus_{i \in I} Y_{i}, \tau, \preceq\right) \rightarrow\left(\oplus_{i \in I} Z_{i}, \theta, \leq\right)$ is $I$ (resp. $\left.D, B\right)$-continuous if and only if every mappings $f_{i}:\left(Y_{i}, \tau_{i}, \preceq_{i}\right) \rightarrow\left(Z_{i}, \theta_{i}, B, \leq_{i}\right)$ is I (resp. D, B)-continuous.

Proof. We prove the theorem in the case of I-continuity and one can prove the cases between parentheses similarly.

Necessity: Suppose that a mapping $f:\left(\oplus_{i \in I} Y_{i}, \tau, \preceq\right) \rightarrow\left(\oplus_{i \in I} Z_{i}, \theta, \leq\right)$ is I-continuous. Taking an arbitrary map $f_{j}:\left(Y_{j}, \tau_{j}, \preceq_{j}\right) \rightarrow\left(Z_{j}, \theta_{j}, \leq_{j}\right)$, where $j \in I$. Let $G$ be an open subset of $\left(Z_{j}, \theta_{j}, \leq_{i}\right)$. Then $G$ is an open subset of $\left(\oplus_{i \in I} Z_{i}, \theta, \leq\right)$. By assumption, $f^{-1}(G)$ is an increasing open subset of $\left(\oplus_{i \in I} Y_{i}, \tau, \preceq\right)$. Since $G \cap Z_{i}=\varnothing$ for each $i \neq j$, then $f^{-1}(G)=f_{j}^{-1}(G)$. Therefore $f_{j}^{-1}(G)$ is an increasing open subset of $\left(Y_{j}, \tau_{j}, \preceq_{j}\right)$, as required.

Sufficiency: Suppose that $f_{i}:\left(Y_{i}, \tau_{i}, \preceq_{i}\right) \rightarrow\left(Z_{i}, \theta_{i}, \leq_{i}\right)$ is I-continuous for every $i \in I$ and let $H$ be an open subset of $\left(\oplus_{i \in I} Z_{i}, \theta, \leq\right)$. Now, $H \cap Z_{i}$ is an open subset of $\left(Z_{i}, \theta_{i}, \leq_{i}\right)$ for every $i \in I$. By assumption, $f_{i}^{-1}\left(H \cap Z_{i}\right)$ is an increasing open subset of $\left(Y_{i}, \theta_{i}, \preceq_{i}\right)$ for every $i \in I$. Therefore $\bigcup_{i \in I} f_{i}^{-1}\left(H \cap Z_{i}\right)$ is an increasing open subset of $\left(\oplus_{i \in I} Y_{i}, \tau, \preceq\right)$. Since $f^{-1}(H)=$ $\bigcup_{i \in I} f_{i}^{-1}\left[\left(H \cap Z_{i}\right)\right.$, then $f^{-1}(H)$ is an increasing open subset of $\left(\oplus_{i \in I} Y_{i}, \tau, \preceq\right)$, as required.

In a similar way, one can prove the following three results.

Theorem 3.4. A mapping $f:\left(\oplus_{i \in I} Y_{i}, \tau, \preceq\right) \rightarrow\left(\oplus_{i \in I} Z_{i}, \theta, \leq\right)$ is $I$ (resp. $\left.D, B\right)$-open if and only if every mappings $f_{i}:\left(Y_{i}, \tau_{i}, \preceq_{i}\right) \rightarrow\left(Z_{i}, \theta_{i}, \leq_{i}\right)$ is $I$ (resp. $D, B$ ) -open.

Theorem 3.5. A mapping $f:\left(\oplus_{i \in I} Y_{i}, \tau, \preceq\right) \rightarrow\left(\oplus_{i \in I} Z_{i}, \theta, \leq\right)$ is $I$ (resp. $\left.D, B\right)$-closed if and only if every mappings $f_{i}:\left(Y_{i}, \tau_{i}, \preceq_{i}\right) \rightarrow\left(Z_{i}, \theta_{i}, \leq_{i}\right)$ is $I$ (resp. $\left.D, B\right)$-closed. 
Corollary 3.2. A mapping $f:\left(\oplus_{i \in I} Y_{i}, \tau, \preceq\right) \rightarrow\left(\oplus_{i \in I} Z_{i}, \theta, \leq\right)$ is $I$ (resp. $\left.D, B\right)$-homeomorphism if and only if every mappings $f_{i}:\left(Y_{i}, \tau_{i}, \preceq_{i}\right) \rightarrow\left(Z_{i}, \theta_{i}, \leq_{i}\right)$ is I (resp. D, B) -homeomorphism.

The other path of this study is the answer of the following two questions:

(1) Under what conditions a topological ordered space represents the sum of topological ordered spaces?

(2) If a topological ordered space represents the sum of topological ordered spaces, what is the maximum number of these topological ordered spaces?

The following results answer these questions.

Theorem 3.6. If $(Y, \tau, \preceq)$ is order-separated, then it represents the sum of two topological ordered spaces.

Proof. Since $(Y, \tau, \preceq)$ is order-separated, then it contains at least a proper monotonically clopen set $X$. Therefore the two subspaces $\left(X, \tau_{X}, \preceq_{X}\right)$ and $\left(X^{c}, \tau_{X^{c}}, \preceq_{X^{c}}\right)$ are topological ordered spaces such that $(Y, \tau, \preceq)$ is their sum.

Theorem 3.7. If $Y=\bigcup_{i \in I} Y_{i}$ such that all $Y_{i}$ are monotonically clopen subset of $(Y, \tau, \preceq)$. Then the maximum partition of $\left\{Y_{i}: i \in I\right\}$ for $Y$ represents the maximum number of topological ordered spaces such that $(Y, \tau, \preceq)$ is their sum.

Proof. Straightforward.

\section{Conclusion}

Topological ordered space combines of two mathematical structures: topology and partial order relation on the universal set such that topology and partial order relation are defined as independent each of other. However, the interaction between them occurs in the case of defining some concepts using some characteristics of topology and partial order relation such as monotonic open (closed) sets and the smallest or largest element of some $T_{i}$-ordered spaces. It can be observed on classical topology that there exists an open set containing an element if and only if there exists a neighborhood of this element. This leads to the equivalence of using open sets or neighborhoods of defining some topological concepts such as $T_{i}$-spaces. On the other hand, it can be noted on ordered topology that a monotonic neighborhood need not be a monotonically open neighborhood. Therefore we obtain $T_{i}$-ordered spaces using monotonic open sets different than their counterparts using monotonic neighborhoods.

In this article, we have introduced and studied sum of the ordered spaces using pairwise disjoint topological ordered spaces. We have defined ordered additive, finitely ordered additive and countably ordered additive properties and then investigated some concepts with respect to these properties. If we consider a partial order relation is the equality relation we note that the properties and findings concerning sum of the spaces given in [17] are special cases of the properties and findings obtained in this work.

We plan in an upcoming paper to introduce and explore the concept of sum of the spaces on soft ordered setting. 


\section{Acknowledgments}

The author would like to thank the referees for their valuable comments and suggestions which help to improve the manuscript.

\section{References}

[1] M. Abo-Elhamayel and T. M. Al-shami, Supra homeomorphism in supra topological ordered spaces, Facta Univ. Ser. Math. Inform., 31 (5) (2016) 1091-1106.

[2] T.M. Al-shami, Compactness on ordered setting with application on the information system, Submitted.

[3] T. M. Al-shami and M. E. El-Shafei, On supra soft topological ordered spaces, Arab Journal of Basic and Applied Sciences, 26 (1) (2019) 433-445.

[4] T. M. Al-shami and M. E. El-Shafei, Some types of soft ordered maps via soft pre open sets, Applied Mathematics \& Information Sciences, 13(5) (2019) 707-715.

[5] T. M. Al-shami and M. E. El-Shafei, Two new forms of ordered soft separation axioms, Demonstratio Mathematica, 53 (2020) 8-26.

[6] T. M. Al-shami, M. E. El-Shafei and M. Abo-Elhamayel, On soft topological ordered spaces, Journal of King Saud University-Science, 31 (4) (2019) 556-566.

[7] T. M. Al-shami, M. E. El-Shafei and M. Abo-Elhamayel, On soft ordered maps, General letters in Mathematics, 5(3) (2018) 118-131

[8] T. M. Al-shami, M. E. El-Shafei and M. Abo-Elhamayel, New types of soft ordered mappings via soft $\alpha$-open sets, Italian Journal of Pure and Applied Mathematics, 42 (2019) 357-375.

[9] T. M. Al-shami, M. E. El-Shafei and B. A. Asaad, Other kinds of soft $\beta$ mappings via soft topological ordered spaces, European Journal of Pure and Applied Mathematics, 12 (1) (2019) 176-193.

[10] T. M. Al-shami, L. D. R. Kočinac and B. A. Asaad, Sum of soft topological spaces, Mathematics, 8(6) 2020, 990; doi:10.3390/math8060990.

[11] S. D. Arya and K. Gupta, New separation axioms in topological ordered spaces, Indian J. Pure Appl. Math., 22(1991), 461-468

[12] D. C. J. Burgess and M. Fitzpatrick, On separation axioms for certain types of ordered topological space, Math. Proc. Camb. Phil. Soc., 82 (1) (1977), 59-65.

[13] D. C. J. Burgess and S. D. McCartan, Order-continuous functions and order-connected spaces, Proc. Camb. Phil. Soc., 68 (1970), 27-31.

[14] P. Das, Separation axioms in ordered spaces, Soochow Journal of Mathematics, 30 (2004) 447-454.

[15] M. E. El-Shafei, M. Abo-Elhamayel and T. M. Al-shami, Strong separation axioms in supra topological ordered spaces, Mathematical Sciences Letters, 6 (3) (2017), 271-277.

[16] M. E. El-Shafei and T. M. Al-shami, Some new types of soft b-ordered mappings, International Journal of Advances in Mathematics, Volume 2019 (3) (2019) 1-14.

[17] R. Engelking, General Topology, Heldermann, Berlin, 1989.

[18] M. K. R. S. V. Kumar, Homeomorphism in topological ordered spaces, Acta Ciencia Indica, XXVIII(M)(1)(2012) 67-76.

[19] H.-P. A. Künzi, Completely regular ordered Spaces, Order, 7 (1990) 283-293.

[20] H.-P. A. Künzi and T. A. Richmond, $T_{i}$-ordered reflections, Applied General Topology, 6 (2) (2005) 207-216.

[21] H.-P. A. Künzi and T. A. Richmond, Completely regularly ordered spaces versus $T_{2}$-ordered spaces which are completely regular, Topology and its applications, 135 (2004) 185-196.

[22] S. Lazaar and A. Mhemdi, On some properties of $T_{0}$-ordered reflection, Applied General Topology, 15 (1) (2014) 43-54.

[23] D. S. Leela and G. Balasubramanian, New separation axioms in ordered topological spaces, Indian Journal of Pure and Applied Mathematics, 33 (2002) 1011-1016.

[24] S. D. McCartan,On subsets of a partially ordered set. Proc. Cambridge Philos. Soc. 62 (1966), 583-595. 
[25] S. D. McCartan, Separation axioms for topological ordered spaces, Math. Proc. Camb. Philos. Soc., 64 (1968) 965-973

[26] S. D. McCartan, Bicontinuous preordered topological spaces, Pacific J. Math., 38 (1971) 523-529.

[27] O. Mendez, L. H. Popescu and E. D. Schwab, Inner separation structures for topological spaces, Balkan J. Geom. Appl., 13 (2)(2008) 59-65.

[28] L. Nachbin, Topology and order, D. Van Nostrand Inc. Princeton, New Jersey, 1965.

[29] L. Popescu, R-Separated spaces, Balkan J. Geom. Appl., 6 (2)(2001) 81-88.

[30] S. Shanthi and N. Rajesh, Separation axioms in topological ordered spaces, Italian Journal of Pure and Applied Mathematics, 40 (2018) 464-473. 